山्山FANÇAISE

$>\mathrm{DE}$

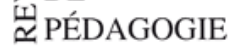

Revue française de pédagogie

Recherches en éducation

$197 \mid 2016$

Les multiples facettes de la créativité dans

l'apprentissage

\title{
Créativité et réflexivité : vers une démarche innovante de formation des enseignants
}

Creativity and reflexivity: towards an innovative approach to teacher training

Isabelle Capron Puozzo et Bernard Wentzel

\section{(2) OpenEdition}

\section{Journals}

Édition électronique

URL : https://journals.openedition.org/rfp/5147

DOI : $10.4000 /$ rfp.5147

ISSN : 2105-2913

Éditeur

ENS Éditions

Édition imprimée

Date de publication : 31 décembre 2016

Pagination : $35-50$

ISSN : 0556-7807

Référence électronique

Isabelle Capron Puozzo et Bernard Wentzel, « Créativité et réflexivité : vers une démarche innovante de formation des enseignants », Revue française de pédagogie [En ligne], 197 | 2016, mis en ligne le 31 décembre 2019, consulté le 07 janvier 2022. URL : http://journals.openedition.org/rfp/5147; DOI https://doi.org/10.4000/rfp.5147 


\title{
Créativité et réflexivité : vers une démarche innovante de formation des enseignants
}

\author{
Isabelle Capron Puozzo \\ Bernard Wentzel
}

\begin{abstract}
La créativité est devenue une capacité de plus en plus présente au sein des curricula scolaires comme, de manière plus récente, au sein des référentiels de compétence de formation des enseignants. Alors que la figure du praticien réflexif est déjà bien intégrée comme développement d'une compétence professionnelle, elle cherche encore des voies innovantes de développement. Cet article tisse une réflexion théorique, tout d'abord, sur l'articulation possible entre la créativité et la réflexivité tant sur le plan conceptuel que sur un plan plus pragmatique à travers l'écriture. Dans un second temps, sur la base de données empiriques recueillies au sein d'une formation intercantonale en Suisse, il s'agit de comprendre par l'analyse si l'écriture créative est une piste favorable au développement de la pratique réflexive. Les résultats montrent que, si la production écrite ne révèle pas toujours les traces d'une créativité ou d'une réflexivité, l'analyse des métatextes met en évidence que le processus, décrit dans ces derniers, a souvent été créatif et très réflexif. L'article s'achève autour d'une réflexion sur l'autofiction comme piste nouvelle pour développer la créativité et la réflexivité.
\end{abstract}

Mots-clés (TESE) : créativité, formation des enseignants, exercice écrit, innovation pédagogique

\section{Introduction}

Former les futurs enseignants à être des praticiens réflexifs relève d'un défi permanent pour les formateurs. À partir de différentes perspectives théoriques, I'opérationnalisation de formations à la pratique réflexive continue à donner lieu à diverses innovations. Les formateurs d'enseignants sont par exemple amenés à proposer un enseignement créatif (Craft, 2005) en introduisant régulièrement des tâches nouvelles. Néanmoins, des nouvelles tâches n'impliquent pas dans tous les cas de promouvoir l'apprentissage créatif (Craft, 2005) des étudiants.

Si les travaux menés sur l'analyse de pratiques professionnelles (voir par exemple Altet, 2000; Altet, Desjardins, Étienne et al., 2013) ont pu mettre en évidence des 
effets de formation à la réflexivité chez les futurs enseignants, l'innovation pédagogique dans l'enseignement supérieur consiste à envisager de nouveaux chemins pour mieux répondre à des objectifs de formation et de professionnalisation de plus en plus complexes, mais en adéquation avec la vision systémique du monde actuel (Craft, 2005; Robinson, 2011). De nombreux travaux scientifiques (Marcel, Dupriez, Perisset Bagnoud et al., 2007; Tardif, Marcel, Perisset et al., 2017) traitent de cette complexité croissante et en esquissent certains facteurs : l'évolution des formes d'organisation et de division du travail avec la collaboration et le travail en équipe en ligne de mire, l'inflation des savoirs à enseigner, la nécessité de former des professionnels pouvant répondre aux défis d'une école intégrative et connectée autant aux nouvelles technologies qu'au monde de l'entreprise, etc. La formation professionnelle s'empare donc de cette complexité pour préparer ces acteurs à relever de nombreux défis, par leur engagement et leur réflexivité d'acteurs critiques, par leur expertise et leur polyvalence, par leur créativité et leurs capacités à innover. Penser l'innovation pédagogique (Cros, 2002, 2007, 2013) induit de facto d'être tout d'abord dans une démarche créative. En effet, ce sont les processus créatifs qui sont à la base de l'innovation (Csikszentmihalyi, 2006), également présente dans la formation professionnelle. Raison de plus pour que l'innovation s'invite en formation, en Suisse, dans des institutions tertiaires/ universitaires telles que les Hautes écoles pédagogiques qui stabilisent petit à petit leur identité, entre recherche, action, théorie, pratique, formateurs, enseignants et chercheurs.

En s'appuyant sur des fondements psychologiques de la créativité (Lubart, 2010), sur des théories issues des sciences de l'éducation sur la créativité (Craft, 2005 ; Capron Puozzo, 2016a) et sur la réflexivité (Altet, Desjardins, Étienne et al., 2013; Morisse \& Lafortune, 2014; Tardif, Borgès \& Malo, 2012; Schön, 1983), cet article repose sur l'analyse de 14 productions d'étudiants, élaborées à partir d'une technique d'écriture créative, sur la volée 2015-2016 de la Formation intercantonale romande des professionnels de l'enseignement des activités créatrices, de l'éducation nutritionnelle et de l'économie familiale (Piracef)'. Cette étude de cas d'un artefact - les récits créatifs (Albarello, 2011) - repose sur l'hypothèse que la créativité peut potentiellement développer la réflexivité : il s'agit

1 Voir en ligne : <https://www.piracef.ch> (consulté le 15 juin 2016). d'identifier les indices dans les productions qui manifestent que les étudiants ont non seulement été créatifs, mais aussi réflexifs.

En introduisant préalablement un cadre conceptuel qui esquisse les bases possibles - essentiellement sous forme d'hypothèse de travail ici - de rapprochements entre créativité et réflexivité, nous explorons donc ce croisement dans l'analyse des productions d'étudiants. Les résultats présentés dans cet article mettent en évidence des perspectives intéressantes pour préciser, approfondir, au niveau conceptuel, certaines formes de similitude.

\section{Cadre conceptuel}

\section{La créativité : définition et enjeux}

Dans une perspective de psychologie différentielle, cet article s'appuie sur une définition consensuelle de la créativité comme "production nouvelle et adaptée» (Lubart, 2010, p.10) au contexte dans lequel elle se manifeste. Cette production peut à la fois prendre la forme d'un produit (une production textuelle, une maquette, un dessin, une performance artistique, la résolution d'un problème scientifique, etc.) ou d'un microprocessus cognitif tel que la pensée divergente/ convergente (capacité à générer le plus d'idées et à retenir la plus pertinente), la comparaison ou combinaison sélective (l'association de plusieurs idées appartenant à des domaines proches ou lointains), la flexibilité, etc. (Lubart, 2010). Une production est l'aboutissement d'une idée générée par plusieurs microprocessus cognitifs. Cette définition soulève la tension entre le processus mené, qui a peut-être été créatif, et la production effective, qui ne l'est quant à elle peut-être pas. Ainsi, dans le cadre de la recherche en éducation, il est intéressant de tenter d'identifier cette créativité aussi bien dans le produit, comme une production écrite, que dans le processus par des entretiens, des vidéos ou des productions invitant explicitement à verbaliser ce dernier (à titre d'exemple, des méta-textes)².

Mais comment identifier ces traces de la créativité? Pour ce faire, la notion d'adaptation au cœur de la définition de la créativité implique qu'un cadre, bien défini

2 Le méta-texte est un texte réflexif dans lequel l'étudiant fait le bilan de sa formation en pointant le développement de compétences professionnelles tant sur l'appropriation des savoirs que sur leur mise en pratique sur le terrain. II se réfère à l'ensemble des textes produits tout au long de la formation. 
par des contraintes, est nécessaire pour que cette créativité ne devienne pas un processus anarchique (Sternberg \& Kaufman, 2010). En effet, la créativité n'est pas synonyme de liberté et d'absence de contrainte (Boden, 2001). L'Ouvroir de littérature potentielle, plus communément connu sous l'acronyme Oulipo, est un exemple significatif de l'importance de la notion de contrainte pour le développement de la créativité dans l'écriture (Desmée, 2009). Certes, la créativité exprime la liberté de générer des idées, d'explorer des chemins différents, de proposer plusieurs solutions, mais toujours au sein d'un cadre bien défini. C'est dans ce cadre uniquement, délimité par une ou plusieurs contraintes, que l'enseignant crée dès lors un espace pour repousser les limites, pour approfondir des idées nouvelles et variées et pour ne pas avoir une seule et unique solution. De même, pour le chercheur, ce cadre lui permet d'analyser la créativité. Sinon, la production est considérée comme hors-sujet (Besançon \& Lubart, 2008). La contrainte ouvre donc des possibles, ce que Bonnardel appelle «l'ouverture de l'espace de recherche d'idées » (2016, p. 170). Dans le contexte éducatif, élaborer une tâche ayant pour objectif explicite de développer la créativité des élèves (Craft, 2005) et d'ouvrir, en conséquence, à cette recherche d'idées, implique une réflexion sur le type de contraintes suffisamment complexes qui va favoriser le processus créatif.

Une fois cet espace ouvert, il n'est pas garanti que l'apprenant s'investisse dans un tel processus d'exploration des possibles. II est plus rassurant de n'avoir qu'une seule solution à chercher et les élèves plus âgés ou les adultes en formation sont souvent plus déstabilisés que rassurés par de tels dispositifs d'apprentissage (Capron Puozzo, 2016a). D'où l'intérêt de construire une école qui intègre la créativité dès la petite enfance et tout au long de la scolarité et de la formation (Capron Puozzo, 2016b). Déjà au début du XX $X^{\mathrm{e}}$ siècle, les travaux de Vygostki ([1930] 2004) révèlent que la courbe du développement de l'imagination diminue avec le développement d'une pensée conceptuelle complexe au moment de l'adolescence. Il est ainsi nécessaire de ne pas laisser la créativité être broyée par la connaissance (Boden, 2001) et de réfléchir à des pistes d'action pour développer continuellement l'imagination (ClercGeorgy, 2016) et, en conséquence, la créativité. «Nurture the knowledge without killing creativity» (Boden, 2001, p. 102). Dans un tel projet, l'écriture apparaît dès lors comme une piste propice et fertile pour faire jaillir, faire émerger et valoriser cette créativité.

\section{Quelques mots sur la réflexivité}

Avant de soutenir la thèse de cette relation possible entre créativité et réflexivité - dans un premier temps à un niveau conceptuel puis à l'épreuve de l'empirie -, commençons par poser quelques jalons. Guillemette (2016) a proposé un panorama qui nous semble intéressant de l'exploitation du concept de pratique réflexive au cours du XXe siècle, de Dewey à Vermersch, en passant par Schön, Lewin ou encore Argyris. Comme pour la créativité, la pratique réflexive est usitée communément dans la formation professionnelle, mais avec des «conceptions » différentes (Guillemette, 2016, p. 1). Il est, en effet, incontestable que le paradigme réflexif est devenu l'un des fers de lance du processus de professionnalisation de l'enseignement, de la délimitation de professionnalités (Paquay, 1994) à la référentialisation des compétences, en passant par l'innovation en formation pour opérationnaliser un modèle du praticien réflexif (Altet, 2013). Il est tout aussi incontestable que ce modèle s'est « dilué et fragmenté au fur et à mesure de sa diffusion» (Tardif, Borgès \& Malo, 2012, p. 12).

De ce point de vue, les essais de mise en relation entre créativité et réflexivité pourraient constituer une tentative de plus de reconstruction et de distorsion risquant de diluer encore un peu plus ce «quasiparadigme» réflexif (Tardif, Borgès \& Malo, 2012, p. 12). Le paradigme du praticien réflexif positionne l'enseignant - avec les contrastes voire les limites que cela implique - comme acteur principal de la construction de ses propres savoirs professionnels. Cette perspective constructiviste est à mettre en relation avec les remises en question d'un modèle des sciences appliquées, notamment dans les travaux de Schön (1983, 1994). Le savoir généralisé et le savoir technique ne peuvent suffire à constituer les bases de l'exercice d'une profession. C'est ce que rappelait justement Saint-Arnaud (2001) lorsqu'il parlait du malaise des praticiens tentant d'appliquer les modèles et les théories appris en formation initiale.

Lorsqu'on parle de réflexivité, le processus en jeu est basé sur un retour en arrière : «la réflexion est considérée comme un retour de la pensée sur elle-même en vue d'examiner et d'approfondir un de ses actes spontanés ou un ensemble de ceux-ci » (Blay, 2003, cité dans Morisse, 2014, p. 32). Il est bien question ici de continuité de l'expérience ou, plus précisément, de «travail particulier sur l'expérience ordinaire, un travail psychologique et cognitif» (Piot, 2012, p.99). 
La définition de la réflexivité que propose Altet semble constituer une synthèse accessible de ces différents éléments. En effet, elle la définit comme une «posture» $(2013$, p. 42) de décentration par rapport à l'agir professionnel en vue de la construction d'une pratique professionnelle fondée continuellement sur le questionnement.

Dans une perspective proche, Chaubet rappelle précisément que la visée essentielle de la pratique réflexive, selon Dewey, est «que l'apprenant utilise intelligemment l'expérience actuelle pour ouvrirl'accès à des expériences à venir. Cela augmentera considérablement ses chances de se retrouver moins démuni face aux situations inédites à venir» (2010, p. 195). La place et l'aide apportée par des outils conceptuels (comme par exemple les savoirs issus de la recherche) dans le travail sur l'expérience apparaissent de manière explicite dans diverses pratiques réflexives. La contrainte du détour théorique sur l'expérience ouvre des possibles, un espace de recherches d'idées pour reprendre des termes spécifiques à la créativité. C'est d'ailleurs chez Dewey qu'apparaît l'esquisse de ces liens possibles entre réflexivité et créativité. Piot soulève que la démarche d'enquête réflexive chez Dewey s'appuie sur le déjà-là, c'est-à-dire les expériences antérieures, pour produire une pensée créatrice, des résultats nouveaux qui vont être intégrés à un système de résolution de problème pour agir (2012, p.97). Nous retrouvons également cette dimension d'une pensée réflexive/créatrice chez Schön, comme le relèvent justement Saussez, Ewen et Girard : «le praticien est un artiste dans le sens où il crée des solutions originales (et toujours provisoires) en réponses à des situations problématiques. Ces réponses originales, même si elles utilisent, parfois, des connaissances scientifiques comme outils, ne constituent pas pour autant une simple application de ces connaissances » $(2001$, p. 75$)$. La création, dans les termes de Dewey (1936), est bien portée par cette idée de résolution de problèmes. Néanmoins, la place d'outils conceptuels dans le travail sur l'expérience est essentielle. Notons que la notion d'enquête renvoie ici à une posture de recherche sans être pour autant, exclusivement, à comprendre comme une démarche scientifique. Perrenoud parle de posture quotidienne d'être en recherche, de l'ordre de dispositions intériorisées, parmi lesquelles des compétences, mais aussi un rapport réflexif au monde et au savoir, une curiosité, un regard distancié, des attitudes, l'envie de comprendre» (2001, p. 76). Cette décentra- inversement, cette capacité à se remettre en question et à envisager une même situation sous plusieurs angles impliquent qu'une démarche réflexive est indubitablement liée à la flexibilité et à la divergence créative. Comme pour la créativité, la réflexivité peut être identifiée aussi bien dans des productions (écrites ou orales) que dans le processus verbalisé (à l'écrit ou à I'oral) et qui amène à convoquer les savoirs théorique et pratique.

\section{Créativité, réflexivité et écriture}

L'écriture, non pas comme production universitaire canonique, mais comme démarche créative, constitue une forme d'opérationnalisation à la fois pour le développement de la créativité mais aussi pour celui de la réflexivité, particulièrement dans le champ de la formation. Elle permet ainsi de mettre en synergie deux compétences nécessaires aux professionnels de l'enseignement et de proposer une piste concrète de rapprochement.

\section{Écriture et créativité}

L'écriture littéraire est un univers intrinsèquement créatif (Woods, 2001). Même lorsqu'une histoire est revisitée, l'auteur exprime indubitablement sa créativité en proposant de la nouveauté, que ce soit au niveau de la diégèse (I'univers du récit; Genette, 1972) ou de la linguistique. Le pouvoir démiurgique ${ }^{3}$ des mots constitue un puissant catalyseur de l'imagination. Lapaire parle, de manière plus générale, d'une «pédagogie de la démiurgie» $(2016$, p. 7$)$ que l'on pourrait dès lors encourager à l'école ou en formation pour stimuler le processus créatif.

Cependant, dans le contexte de l'éducation, comment l'écriture peut-elle être aussi bien un moyen pour développer la créativité qu'un outil pour questionner l'expérience et s'approprier les savoirs?

En effet, en situation d'apprentissage, développer la créativité est un objectif transversal qui pourrait être atteint tout en visant l'appropriation de savoirs théoriques dans le but de les mettre en œuvre dans la pratique. L'écriture devient dès lors « un outil de médiation au service du développement des savoirs professionnels » (Cros, 2004, p. 132). En référence aux travaux de Cifali (1996), Cros (2004) montre que l'écriture permet : 1) de s'approprier un métalangage, 2) à la fois de se

3 La démiurgie désigne le pouvoir créateur. 
rapprocher et de se distancier du savoir d'action et 3) de favoriser la réflexivité sur l'action. De plus, la créativité dans la littératie, et plus spécifiquement l'écriture, est un bon moyen pour développer la motivation à écrire (Vanasse \& Gaudreault, 2004), car elle offre un mode d'expression écrite différent des productions habituelles. Face à un public résistant aussi bien à l'écriture qu'à l'appropriation de notions conceptuelles, la créativité dans l'écriture ouvre donc une voie fertile pour développer la motivation à apprendre (Viau, 2009) tout en favorisant la réflexivité des enseignants qui ne peut s'opérer sans la maîtrise du savoir théorique.

\section{Écriture réflexive et professionnalisation}

L'engouement qu'a suscité le paradigme réflexif, particulièrement suite aux travaux de Schön (1983, 1994), a influencé l'émergence de nouvelles conceptions de la formation professionnelle, plus analytiques et distantes face à la construction des savoirs théoriques et pratiques. Pour ce faire, l'écriture s'est ouverte comme une piste fertile, mais néanmoins difficile à apprivoiser pour les étudiants. En effet, Crinon et Guigue rappellent justement que l'entrée dans l'écriture, en contexte de formation, est un exercice délicat qui pose notamment la question des genres discursifs à disposition des auteurs : «la nécessité d'articuler les différentes dimensions (le vécu et les connaissances, le professionnel et les savoirs acquis), d'éviter la simple narration comme le discours théorique expositif général» (2006, p. 128) peut être vécue comme une injonction paradoxale. La construction de ces genres s'avère complexe, comme le soulignent les auteurs qui en passent en revue un certain nombre tels le mémoire professionnel, le portfolio, le journal de formation ou encore les histoires de vie. Il convient donc d'instrumenter l'entrée dans la discursivité pour favoriser des démarches réflexives diversifiées. Pour Vanhulle, «cette instrumentation doit pouvoir favoriser l'investissement de l'étudiant dans la discursivité, dans l'exploration du langage au service d'une pensée critique et créative davantage que la seule maîtrise de "techniques" d'écriture réflexive» (2005, p.61). Nous pouvons prolonger ici la question formulée dans le paragraphe précédent : en quoi l'écriture favorise-t-elle I'activité réflexive et de professionnalisation (Morisse, 2014) ? Tout d'abord, selon Morisse (2014), l'écriture est un outil de prise de conscience. Par rapport à une action passée, elle permet de s'emparer de ce que l'on fait et de ce que l'on sait, de ce qui s'éloigne du prescrit, au service de la construction de nouveaux savoirs. Néanmoins, lorsqu'on parle d'écriture réflexive, il ne s'agit pas de se limiter à décrire les faits, à restituer une action. Morisse propose de questionner le récit comme outil de distanciation, de saisie de l'action vécue pour la placer à l'extérieur de soi : «l'art de raconter, par l'intermédiaire du travail de composition, favorise ainsi la fiction et la distanciation par rapport au texte (2014, p. 19). Ensuite, selon un principe dynamique qui associe l'écriture et l'activité cognitive, l'écriture réflexive permet d'établir une médiation «entre l'auteur et luimême, l'amenant à préciser, structurer ou épaissir le texte produit, par des retours récursifs, favorisant un travail d'élucidation, de clarification et d'élaboration de sa propre pensée» (Morisse, 2014, p. 21). Elle est, par là même, un outil au service de la construction de ses propres connaissances. Enfin, relevons encore chez Morisse la dimension dialogique tout à fait intéressante de l'écriture réflexive qui nourrit le dialogue avec différents interlocuteurs, tout autant que la rencontre avec d'autres auteurs (intertextualité).

En conséquence, il devient intéressant d'explorer en formation différents types de production textuelle qui concourent à développer l'identité professionnelle. La recherche présentée ouvre un espace de réflexion autour d'une production qui permet de développer l'écriture créative tout en convoquant le savoir théorique de formation.

\section{Enquête sur la créativité et la réflexivité dans la formation intercantonale}

\section{Contexte et description du dispositif de formation}

\section{La formation Piracef}

Le contexte dans lequel s'inscrit cette recherche est celui de la Formation intercantonale romande des professionnels de l'enseignement des activités créatrices (AC), de l'éducation nutritionnelle (EN) et de l'économie familiale (EF) (Piracef) en Suisse ${ }^{4}$. Les étudiants sont en cours d'emploi visant la titularisation par cette formation sur les postes d'enseignants d'activités

4 Pour approfondir d'autres résultats de recherche menés au sein de cette formation, voir Bonnardel \& Didier, 2016; Didier, 2016; Didier, Perrin \& Vanini De Carlo, 2016; Leuba, Didier, Perrin et al., 2012. 
créatrices et manuelles et d'économie familiale. La formation vise à obtenir 40 crédits en 3 années. Le plan de formation comprend des modules obligatoires et optionnels en didactique, en sciences de l'éducation, dans la discipline (par exemple, pâtisserie, cuisine pour $E F$, matériaux souples, travail en 3D pour $A C$ ) et en pratique réflexive (séminaire d'intégration). La recherche menée a été effectuée dans la filière Économie familiale, durant le séminaire obligatoire d'intégration.

Ce séminaire se déroule durant les 3 années à hauteur d'un crédit par an avec le suivi d'un même groupe par le même formateur. Il est évalué sur la base d'un dossier de formation comportant :

- 6 courts textes d'une à deux pages dans chacun desquels l'étudiant choisit un concept étudié en formation qu'il discute de manière réflexive;

- une écriture créative (la fiction scientifique);

- un méta-texte visant à faire le bilan de la formation.

\section{La fiction scientifique dans le séminaire d'intégra- tion ou l'art d'aborder le savoir de formation en se racontant des histoires}

L'écriture d'une fiction scientifique a été intégrée à la $3^{\mathrm{e}}$ année du programme de la pratique réflexive de la formation Piracef dans le but de répondre à l'objectif de développer la posture du praticien réflexif (voir tableau 1 en annexe). La fiction scientifique n'est pas une histoire ordinaire. Dans cette production, «le texte à produire comporte une fiction (histoire inventée), une narration (situations, personnages fictifs ou mise en scène des protagonistes), et il doit par conséquent distraire le lecteur» (Chartier \& Frier, 2009, p. 157). L'histoire doit être nouvelle. En même temps, c'est un récit qui permet au lecteur de comprendre un sujet. En effet, «c'est aussi un récit qui met en scène et incarne, en plus de la fiction, des idées, un point de vue sur une question, un problème scientifique, et il doit donc également permettre d'instruire le lecteur : l'information scientifique doit y être présente sous différentes formes et l'on doit par conséquent trouver dans cet écrit des extraits du discours scientifique mis en scène : "bribes" de textes (citations), notions, idées reformulées, arguments, courants théoriques, noms d'auteurs, dates, etc., qui vont être injectés au fil de I'histoire» (Chartier \& Frier, 2009, p. 157). II s'agit donc de choisir un concept étudié en formation et de l'intégrer tout au long de l'histoire fictive afin que le lecteur le comprenne. Cette imbrication pourra prendre plusieurs formes : allusions à l'auteur scientifique de réfé- définition du concept choisi au sein même de l'histoire, etc. Il est donc nécessaire à la fois de faire preuve d'imagination et, en même temps, d'instruire le lecteur qui lit le texte. "Ces deux impératifs (distraire et instruire) doivent trouver un équilibre dans le texte» (Chartier \& Frier, 2009, p. 158) afin que l'écriture scientifique soit une production nouvelle et adaptée (Lubart, 2010), une production donc créative. Comme il a été mentionné dans le cadre conceptuel, le praticien réflexif est un enseignant capable d'argumenter ses pratiques didactiques et pédagogiques en s'appuyant sur des concepts, des références scientifiques, des théories, etc. (Schön, 1983, 1994). La formation est une opportunité pour s'approprier ces théories et se construire solidement en tant que professionnel de l'enseignement. L'objectif de l'écriture créative est donc de favoriser, par une activité différente et ludique, la construction solide et efficace des objets de savoir afin de pouvoir réfléchir ensuite, que ce soit dans la pratique ou dans le méta-texte, sur sa transposition dans les pratiques d'enseignement/apprentissage. Sachant que le public Piracef est fortement résistant à ce savoir théorique, il s'agit donc de proposer un dispositif pour accompagner les étudiants (voir synopsis en annexe) dans l'écriture créative et les amener vers un apprentissage créatif. L'analyse des productions est nécessaire pour comprendre comment s'articule le savoir théorique dans cette écriture créative et si cette tâche répond à l'objectif de développer la réflexivité.

\section{Méthodologie, question et corpus de données}

La méthodologie de cette recherche est une démarche d'analyse qualitative de documents écrits considérés comme des artefacts au sein d'un module de formation et qui sont analysés dans le cadre d'une étude de cas (Albarello, 2011). Partant du constat que les étudiants sont peu motivés à écrire (Vanasse \& Gaudreault, 2004), la fiction scientifique apparaît comme une piste fertile de formation pour favoriser l'appropriation des savoirs tout en développant une créativité (Capron Puozzo, 2016a; Vanini De Carlo \& Clerc-Georgy, 2011). Nous faisons I'hypothèse que cette tâche peut potentiellement développer la créativité des enseignants par la stimulation de l'imagination tout en permettant de favoriser la réflexivité.

Les données recueillies pour cet article ont été prises auprès de la volée 2015-2016 du séminaire d'intégration qui se compose de 3 hommes et 15 femmes 
ayant une expérience professionnelle allant d'une année à une trentaine d'années.

Le corpus recueilli pour cet article est constitué de quatorze fictions scientifiques et dix méta-textes ${ }^{5}$ d'étudiants de la filière des activités créatrices et manuelles qui ont accepté que leur production soit analysée. Les productions varient entre 3 et 12 pages, sachant que la moyenne est de 4 pages. Dix productions ont été réalisées individuellement et les quatre autres ont été écrites, comme le dispositif le prévoit, à plusieurs ${ }^{6}$. L'ensemble de ce corpus forme un tout sans distinction de la formatrice en charge du séminaire?

La question de recherche que nous soulevons dans cet article est la suivante : quels sont les indices de créativité et de réflexivité identifiables dans l'écriture de fiction scientifique?

Ainsi, dans une perspective de recherche exploratoire, il s'agit d'identifier les traces d'une écriture créative et d'une réflexion réflexive. Au niveau scientifique, I'objectif est d'approfondir les perspectives de rapprochement entre créativité et réflexivité que nous avons esquissées précédemment.

\section{Cadre d'analyse des données}

Pour ce qui est de la créativité, comme nous l'avons montré dans le cadre conceptuel, le corpus est analysé selon le critère du respect des contraintes. La fiction scientifique a donc 3 contraintes :

1. I'univers fictionnel doit résonner avec l'univers conceptuel;

2. le savoir théorique ne doit pas être plaqué dans le texte, mais intégré finement;

3. le texte doit ainsi «distraire et instruire » le lecteur (Chartier \& Frier, 2009, p. 171).

Les résultats de recherche de Chartier et Frier (2009) montrent la difficulté de répondre aux trois contraintes. Dans le contexte de la formation des futurs

5 Dans le respect de la confidentialité du séminaire d'intégration, seuls les méta-textes des étudiants que le premier auteur de cet article avait en séminaire ont été insérés dans le corpus. Les quatre autres étaient dans un autre séminaire.

6 Les productions individuelles ont été réalisées dans le séminaire du premier auteur de l'article et les productions collectives dans le séminaire d'une autre collègue de la formation.

7 Le classement des productions a été effectué individuellement dans un premier temps par les deux auteurs de l'article. La mise en commun a permis de constater une unanimité tant sur la créativité que sur la réflexivité. Le premier auteur de l'article a donné un séminaire; le second auteur est complètement externe à la formation Piracef. enseignants de l'école pré-scolaire et primaire dans une Haute école pédagogique en Suisse, Vanini De Carlo et Clerc-Georgy (2011) ont également mis en œuvre ce dispositif dans le cadre de la pratique réflexive. Si les résultats de leur recherche aboutissent au même constat d'une hétérogénéité dans la capacité à répondre aux contraintes de l'écriture, il en ressort que l'accompagnement dans l'écriture permet aux étudiants d'intérioriser le savoir et de lui donner du sens. Les productions ont ainsi été classées de la moins adaptée aux contraintes fixées par le genre à celle qui est la plus créative et qui répond, en conséquence, au mieux aux contraintes. Seules les productions présentant également des pistes d'analyse sur la réflexivité sont étudiées. Ce classement reste évidemment discutable. Le tableau qui résume les différentes fictions scientifiques se trouve en annexe.

L'analyse de la réflexivité prolonge celle de la créativité en identifiant et interprétant les traces d'une écriture réflexive dans les productions de fiction scientifique, mais aussi dans les méta-textes intégrés au dispositif visant une transposition dans les pratiques. Le cadre d'analyse retenu se compose des catégories suivantes construites à partir du cadre conceptuel et d'une lecture intégrale des données. Dans le vaste paradigme réflexif, nous avons retenu pour construire ces catégories des concepts visant à rendre intelligibles des liens possibles entre réflexivité et créativité. Il s'agit ainsi d'identifier dans les productions :

- des marqueurs de subjectivité qui indiquent un positionnement en première personne dans le texte où l'étudiant se pose comme sujet du discours pour exprimer sa perception, ses émotions et ses actions;

- des éléments de questionnement notamment présents à travers les phrases interrogatives;

- la mobilisation explicite des savoirs appris pour décrire ou expliquer une situation en formation;

- un discours tourné vers une recherche de solution (Wentzel, 2015).

En analysant les productions de fiction scientifique et les méta-textes, avec l'appui des catégories du cadre d'analyse, nous identifions bien entendu des éléments de convergence que nous allons présenter ci-dessous. Précisons d'emblée, avec les données en notre possession, que la mise en évidence de convergences n'a pas pour ambition ici d'approfondir ou de réinterroger les deux concepts en jeu. Au mieux pouvons-nous présenter certaines similitudes dans les processus et dans les visées, autour de la professionnalisation des personnes. Nous pouvons également montrer que ces 
deux concepts ont le potentiel de se nourrir mutuellement, en termes de posture, de pratique, d'état d'esprit ou de rapport au travail. Ces deux éléments traversent notre analyse. Nous en rendons compte ici en nous limitant à deux des catégories d'analyse, empiriquement fondées.

\section{Résultats de l'analyse des écritures de fiction scientifique : émergence de la créativité et de la réflexivité ?}

\section{Le genre littéraire : tension entre créativité et réflexivité}

Sur les quinze productions analysées, quatre ont été considérées comme inadaptées au genre. En effet, ces quatre productions (voir tableau 2 en annexe) n'instruisent pas explicitement puisque : 1 ) les concepts ne sont ni nommés, ni définis; 2 ) aucune référence aux auteurs n'est présente. Seule la fiction est présente. Par rapport à ce dispositif de formation, l'absence de l'objet de savoir, explicité de manière évidente, est problématique. En effet, en référence aux travaux de Vygotski, Triquet démontre que l'écriture est un puissant catalyseur d'apprentissage, car elle permet, dans un contexte d'apprentissage, l'appropriation, mais aussi la restructuration de la pensée : «le traitement narratif d'un contenu scientifique [...] pouvait permettre d'engager un travail de transformation de connaissances » (2007, p. 111). Son absence explicite laisse supposer l'impossibilité d'être dans cette démarche de transformation. Si les références théoriques ne sont pas visibles, le texte est inadapté au genre. Une production qui «n'est pas adaptée [...] sera considérée comme étrange, bizarre» (Besançon \& Lubart, 2008, p. 244), c'est-à-dire hors sujet dans le contexte d'apprentissage. Elle ne répond pas à la contrainte d'instruire le lecteur. II n'y a pas d'apprentissage explicite (Triquet, 2007).

Néanmoins, cette inadaptation à la contrainte première du genre, qui est de plaire et d'instruire, $n^{\prime}$ induit pas forcément une absence de réflexivité. Prenons le cas de la nouvelle policière de Marco qui raconte l'enquête de l'inspecteur Eugène Crintiff sur le meurtre de Marcel, victime du tueur de glace encore non arrêté. Le savoir n'est pas explicite et très peu présent. II faut connaître les concepts pour les identifier dans le texte. La production reste donc inadaptée. En revanche, dans le méta-texte de l'étudiant, il est question de postures et d'état d'esprit, parfois questionnés, parfois explicités ou illustrés, au service de différentes conceptions compréhension ou à la résolution face aux défis de la formation. L'ingénieur créatif de Marco est «plus tenace et saugrenu dans la réflexion que la plupart de ses collègues ». Marco établit des liens plus explicites qui sous-tendent, selon sa propre expérience, une articulation possible entre posture créative et posture réflexive : «durant la formation nous avons vu plusieurs aspects de la technique mélangés à la créativité. Le modèle du praticien réflexif à la manière du designer était une évidence. [...] Cette analyse de la créativité m'a donné une explication concrète sur les différents processus de réflexion autour de la recherche d'idées liées à la technique. Je ne me rendais pas compte que le "talent créatif" pouvait s'expliquer et que ce processus de recherche d'idées était si complexe». L'articulation entre les deux types de production semble donner l'ébauche d'un transfert possible entre créativité et réflexivité.

\section{La fusion des univers fictionnel et conceptuel : entre difficulté d'être créatif et volonté d'appropriation d'une posture réflexive}

Une deuxième catégorie émerge du corpus des données : une tentative de fusion entre la fiction et le savoir théorique. Prenons la production de Fiona qui raconte l'amitié entre Pétronille l'aiguille et Théophile le fil. Pétronille rêve de connaître l'origine de son innovation. Un matin, elle propose à son ami un voyage dans le passé pour rencontrer ses ancêtres aiguilles. L'objet de savoir «l'aiguille à coudre : histoire et technique ${ }^{8}$, qui est un savoir technique, est très bien décrit, même trop au détriment de la fiction : à peine une page pour la fiction en posant le contexte, quatre pages sur l'aiguille et une page et demie pour conclure I'histoire. Un juste équilibre entre la fiction et l'instruction est à trouver. C'est l'inadaptation du texte en lien avec le genre qu'est la fiction scientifique. Le début et la fin sont imprégnés par une créativité linguistique et de jolis jeux de mots en lien avec l'aiguille à coudre («Pétronille l'aiguille et Théophile le fil », «l'art de tisser des liens»). Le lecteur suit cette rencontre entre Pétronille et Théophile qui s'achève comme un conte : ils se marièrent et eurent beaucoup d'aiguilles et de fils à coudre! Néanmoins, au milieu de ce texte, un placage imposant de connaissances sur l'aiguille à coudre

8 Les futurs enseignants de la formation PIRACEF suivent durant leur parcours des modules techniques tels que la couture ou la menuiserie par exemple au sein desquels ils apprennent des savoirs techniques professionnels. 
apparaît. Toute l'explication n'est donc pas imbriquée au sein de la fiction. Dans sa démarche d'écriture, Fiona se centre davantage sur la formalisation de savoirs à enseigner. Le processus de didactisation est développé autour de I'histoire de deux personnages fictifs et se base principalement sur la convocation de savoirs savants. Il s'agit ici d'une transposition didactique et non d'une démarche réflexive, même si une réflexion sur les savoirs en jeu nourrit l'ensemble de la démarche. Les liens possibles entre cette démarche et une pratique réflexive apparaissent néanmoins dans le métatexte de Fiona lorsqu'elle explicite brièvement le sens de sa démarche de didactisation du savoir : «Mettre en lien la théorie et la pratique m'amène à réorienter mes propres représentations d'un professionnel en activités créatrices. En tant que praticien réflexif, je m'interroge, j'analyse ma méthode d'enseignement actuelle car je transmets mes propres savoirs comme je les ai appris, les jeunes gens que j'ai en face de moi ne sont plus ceux de mon époque, la discipline a évolué. Je mobilise les contenus de cours, je les intègre dans mes leçons et celles-ci deviennent riches et vivantes».

La créativité narrative et linguistique est certes bien présente, mais la production n'est pas adaptée, car elle ne respecte pas la contrainte du croisement entre l'univers fictionnel et conceptuel. En conséquence, la résonance n'est pas pertinente, même s'il se dégage de ce texte, comme de celui Marco, cette volonté d'une posture créative du personnage de Pétronille avec «sa capacité à s'adapter». C'est une représentation évidemment fictive de cette posture voulue créative mais qui n'est pas sans rappeler une certaine dimension d'une posture réflexive. Ces différents éléments, aussi favorables à la thèse d'une convergence entre réflexivité et créativité, ne nous disent rien sur une éventuelle appropriation par les auteurs de ces postures qu'ils illustrent. Autant ils font preuve de créativité dans leur fiction scientifique, autant le lien avec une pratique réflexive, de surcroît à vocation professionnalisante, n'est en rien établi. Toutefois, la fin de l'histoire de Fiona laisse sous-entendre une homologie entre le personnage principal et l'auteure qui était elle-même couturière («Au fil du temps, Pétronille au détour de ses rencontres, de ses allersretours entre la théorie et la pratique de ses prérequis et des savoirs en construction, poussée par la motivation et un besoin d'épanouissement personnel, fit face à toutes nouveautés.»). Elle choisit un objet qu'elle maîtrise déjà. Elle ne sort pas de sa zone de confort. Cependant, ce qui est intéressant, c'est que l'on quitte le registre de la fiction scientifique pour aller vers une autofiction (Gasparini, 2011). Cette manière de parler indirectement de soi est une forme d'écriture créative. Cette production est donc théoriquement certes inadaptée, car elle détourne le genre imposé, mais en même temps fortement créative par ce détour mené et cette distinction de la copie par rapport aux autres.

Pour aller plus loin dans l'analyse d'une fusion entre fiction et concepts théoriques, nous avons pu identifier des références à des savoirs scientifiques, plus ou moins explicites, apparaissant sous différentes formes. Certains écrits habilement structurés autour de cette articulation entre théorie et situation fictive laissent supposer l'amorce d'une démarche réflexive. C'est le cas du travail réalisé par Joseline. En effet, celui-ci porte sur des situations d'enseignementapprentissage, posant les bases d'une écriture à vocation professionnalisante. Ensuite, l'auteure convoque, sans vraiment les développer, différents référents théoriques issus de la pédagogie et de la psychologie de l'éducation et s'y réfère pour décrire les situations qu'elle crée. C'est la transposition de ces référents en savoirs pour enseigner qui pourrait amorcer une démarche réflexive à visée professionnalisante. L'amorce de convocation du savoir théorique appris en formation pour expliquer une situation laisse entrevoir quelques indices sur l'appropriation d'une posture réflexive.

\section{Nouveau et adapté : une démarche créative et réflexive}

L'étudiant fait preuve de créativité à partir du moment où il répond aux contraintes fixées par le genre tout en proposant une production nouvelle. Le texte de Nadia est un cas emblématique. Elle raconte I'histoire de l'invention du stylo Bic. Les références aux auteurs sont bien explicitées. Il y a un double apprentissage dans ce texte: sur les facteurs conatifs et sur l'invention du stylo Bic. L'histoire n'est pas complètement imaginaire, puisqu'elle retrace un fait réel. C'est le personnage fictif de Jojo qui l'est. L'étudiante aurait pu aller plus loin dans l'imagination en lui faisant vivre des étapes imaginaires et fictives. Elle s'appuie sur une histoire authentique qui est documentée sur le plan théorique pour illustrer tant des processus créatifs (cognitifs et conatifs) que les difficultés à mettre en œuvre un produit innovant au sein d'un environnement. La fiction, recomposée autour du personnage de Jojo, est finement articulée avec le savoir (les processus conatifs). Et le lecteur apprend tout au long du 
récit. De même, à la posture créative s'ajoute cette posture réflexive partagée entre un objet de réflexion et une réflexion sur la pratique. Le Jojo de Nadia «prenait souvent des risques» et était "ouvert à de nouvelles expériences », «curieux vis-à-vis du monde extérieur et intérieur. Il vivait des situations nouvelles sans aucune anxiété». Dans le méta-texte, la posture réflexive de Nadia y est parsemée de quelques rapprochements avec son Jojo: "J'accueille et je suis confrontée à rechercher de nouveaux défis, projets, nouvelles idées, façons de faire. J'apprends à faire preuve de fluidité et de flexibilité dans la production d'idées » qui renforce cette articulation entre créativité et posture réflexive sans pour autant que soit évidemment prouvée l'appropriation de l'une et de l'autre en synergie.

\section{Conclusion}

Les textes de fiction scientifique que nous avons analysés ne constituent bien entendu pas, a priori, des écrits réflexifs. D'ailleurs, l'écriture réflexive et l'écriture créative restent fondamentalement différentes, tant sur le fond que sur la forme. Le point de départ des fictions scientifiques n'est pas un retour en arrière, sur une pensée, une action passée, mais l'imagination plutôt que l'expérience vécue pour formaliser, voire consolider des connaissances, en l'occurrence professionnelles. Des savoirs théoriques, lorsqu'ils sont convoqués, étayent des représentations et des conceptions probablement développées autant dans l'imagination que dans l'expérience, les deux étant étroitement liées. Le détour par la fiction plutôt que le retour sur l'action pour nourrir une réflexion créative.

Pour revenir à notre question de départ, on peut dire que l'écriture créative de fiction scientifique, en tant que produit, ne permet pas dans tous les cas de déterminer une appropriation créative des connaissances et de révéler une réflexivité. Pour avoir des résultats plus affinés, c'est le processus qui serait à explorer pour éviter de se centrer uniquement sur le produit : processus d'exploration des possibles et de recherche de solutions multiples tant sur le plan théorique que pratique. La fiction scientifique permet effectivement l'appropriation créative des connaissances, même si le résultat (en termes de production écrite) rend visibles des niveaux hétérogènes d'appropriation. De même, pour ce qui est de la réflexivité, il est nécessaire d'aller explorer les méta-textes pour trouver des indices plus pertinents de cette appropria- produits, pris isolément, ne permettent pas de faire des liens à moins que l'étudiant ne l'ait fait lui-même.

Lorsque les méta-textes fournissent certaines clés de lecture des processus de création de la fiction scientifique, notamment par rapport à la convocation de savoirs théoriques puis par rapport aux liens possibles avec des pratiques professionnelles, ils mettent en évidence des postures réflexives. Il est surtout intéressant de constater que les postures réflexives ne constituent pas une fin en soi, même si elles sont présentées comme une composante de l'identité. Elles s'inscrivent dans une visée professionnalisante. C'est bien là tout l'enjeu et c'est bien ce qui permet de favoriser des allers et retours entre réflexivité et créativité. La créativité peut devenir l'objet de postures réflexives; la réflexivité contribue au développement de compétences professionnelles créatives et à un certain état d'esprit dans son rapport à la pratique. Certaines données nous amènent à ces résultats qui sont néanmoins difficilement généralisables. La piste de l'autofiction scientifique (Gasparini, 2011), comme nouveau genre pour la formation professionnelle, semble être une piste intéressante à explorer pour fusionner à la fois une démarche créative et réflexive. Si des effets de formation professionnalisante sont avérés, il se pose néanmoins la question de la pertinence de multiplier les formes d'écriture. En effet, les travaux de recherche mettent généralement en évidence la complexité et parfois les difficultés à faire entrer des étudiants dans des pratiques d'écriture (Crinon \& Guigue, 2006). Même si l'articulation entre des écrits réflexifs et des écrits créatifs mérite sans aucun doute d'être approfondie, un excès de commande de productions écrites pourrait s'avérer contre-productif, aussi complémentaires soient-elles en termes d'effets de formation.

Isabelle Capron Puozzo

Haute école pédagogique du canton de Vaud (Suisse), Centre de soutien à la recherche isabelle.capron-puozzo@hepl.ch

Bernard Wentzel Institut de recherche et de documentation pédagogique (Suisse) bernard.wentzel@irdp.ch 
Bibliographie

ALBARELLO L. (2011). Choisir l'étude de cas comme méthode de recherche. Bruxelles : De Boeck.

ALTET M. (2000). «L'analyse de pratiques : une démarche de formation professionnalisante? ». Recherche et formation, $n^{\circ} 35$, p. 25-41.

ALTET M. (2013). «Formes de résistance des pratiques de formation d'enseignants à la pratique réflexive et conditions de développement de la créativité ». In M. Altet, J. Desjardins, R. Étienne, L. Paquay \& P. Perrenoud (dir.), Former des enseignants réflexifs: obstacles et résistances. Bruxelles : De Boeck, p.39-60.

ALTET M., DESJARDINS J., ÉTIENNE R., PAQUAY L. \& PERRENOUD P. (dir.) (2013). Former des enseignants réflexifs : obstacles et résistances. Bruxelles : De Boeck.

ASTOLFI J.-P. (2008). La saveur des savoirs. Paris : ESF.

BARBEROUSSE A. (1999). L'expérience. Textes choisis et présentés. Paris : Flammarion.

BESANÇON M. \& LUBART T. (2008). «Psychologie de la créativité : place de la créativité dans les apprentissages». In J. Aden, Apprentissage des langues et pratiques artistiques. Créativité, expérience esthétique et imaginaire. Paris : Éd. Le Manuscrit, p. 243-261.

BODEN M. A. (2001). «Creativity and Knowledge». In A. Craft, B. Jeffrey \& M. Leibling , Creativity in Education. Londres : Continuum, p. 95-102.

BODEN M. A. (2005). The creative mind. Myths and mechanisms. New York: Routledge, $2^{\mathrm{e}}$ éd.

BONNARDEL N. (2016). «Propositions de méthodes d'analyse et de modalités d'assistances pédagogique et informatique aux activités créatives. Illustrations dans le domaine du design ». In I. Capron Puozzo, La créativité en éducation et en formation. Perspectives théoriques et pratiques. Louvain-La-Neuve : De Boeck, p. 167-180.

BONNARDEL N. \& DIDIER J. (2016). «Enhancing creativity in the educational design context: An exploration of the effects of design project-oriented methods on students' evocation processes and creative output». Journal of Cognitive Education and Psychology, vol. 15, n¹, p. 80-101.

CAPRON PUOZZO I. (2016a). "Créativité et apprentissage : un mariage prometteur ». Pedagogia PIU'didattica. Teorie e pratiche educative, vol. 2, $n^{\circ} 1$, p. 1-12.

CAPRON PUOZZO I. (2016b). «On ne badine pas avec la créativité». In I. Capron Puozzo, La créativité en éducation et en formation. Perspectives théoriques et pratiques. LouvainLa-Neuve : De Boeck, p.181-183.

CAPRON PUOZZO I. (2016c). «Du concept de créativité à une pédagogie de la créativité : un défi pour le XxI siècle». In I. Capron Puozzo, La créativité en éducation et en formation. Perspectives théoriques et pratiques. Louvain-LaNeuve : De Boeck, p.13-29.

CHARTIER A. \& FRIER C. (2009). "Petite fabrique de la connaissance : aborder le savoir scientifique en se racontant des histoires». Pratiques, vol. 143-144, p.1-20.
CHAUBET P. (2010). La réflexion, processus déclenché et constructeur : cas d'enseignants de FLS en formation ou en exercice et d'aspirants coopérants internationaux. Thèse de doctorat, sciences de l'éducation, Université de Montréal.

CIFALI M. (1996). «Démarche clinique, formation et écriture». In L. Paquay, E. Charlier \& P. Perrenoud, Former des enseignants professionnels. Quelles stratégies... pour quelles compétences? Bruxelles : De Boeck.

CLERC-GEORGY A. (2016). "L'imagination dans le développement de la créativité et de l'apprentissage ». In I. Capron Puozzo, La créativité en éducation et en formation. Perspectives théoriques et pratiques. Louvain-La-Neuve : De Boeck, p.79-92.

CRAFT A. (2005). Creativity in schools. Tensions and dilemmas. New York : Routledge.

CRINON J. \& GUIGUE M. (2006). «Écriture et professionnalisation ». Revue française de pédagogie, n¹56, p.117-169.

CROS F. (2002). «L'innovation en éducation et en formation: topiques et enjeux». In N. Alter, Les logiques de l'innovation. Paris : La Découverte, p. 211-240.

CROS F. (2004). L'innovation aux risques de son évaluation. Paris : L'Harmattan

CROS F. (dir.) (2007). L'agir innovationnel. Entre créativité et formation. Bruxelles : De Boeck.

CROS F. (2013). «De l'initiative à l'expérimentation : la longue vie du soutien à l'innovation ». Les Sciences de l'éducation - Pour l'Ėre nouvelle, vol. 46, n³, p.63-88. En ligne : <http:// www.cairn.info/revue-les-sciences-de-l-education-pourI-ere-nouvelle-2013-3-page-63.htm> (consulté le 15 janvier 2017).

CSIKSZENTMIHALYI M. ([1996] 2006). La créativité. Psychologie de la découverte et de l'invention. Paris : Robert Laffont.

DESMÉE G. (2009). «De la nature d'un atelier d'écriture en milieu professionnel». Vie sociale, $n^{\circ} 2(2)$, p.53-58. En ligne: <www.cairn.info/revue-vie-sociale-2009-2-page-53.htm> (consulté le 20 novembre 2016).

DEWEY J. (1936). La théorie de l'enquête. Paris : PUF.

DIDIER J. (2016). «Corporéité et créativité, entre traditions et innovations». Revue suisse des sciences de l'éducation, vol. $38, \mathrm{n}^{\circ} 1$, p.73-88.

DIDIER J., PERRIN N. \& VANINI DE CARLO K. (2016). «Créativité et conception. Une Learning Study au service de la transformation de l'enseignement des activités créatrices et manuelles». Formation et pratiques d'enseignement en questions, $\mathrm{n}^{\circ} 1$, p.113-128.

FISHER R. \& WILLIAMS M. (dir.) (2004). Unlocking creativity. New York : David Fulton Publishers.

GASPARINI P. (2011). "Autofiction vs autobiographie». Tangence, $n^{\circ} 97$, p.11-24. En ligne : <http://id.erudit.org/ iderudit/1009126ar> (consulté le 12 décembre 2016).

GENETTE G. (1972). Discours du récit, essai de méthode, Figures III. Paris : Éd. du Seuil. 
GUILLEMETTE F. (2016). «Introduction : La pratique réflexive, tout le monde en parle, mais...» Approches inductives, vol. 3, n¹, p.1-6.

HENSLER H., GARANT C. \& DUMOULIN M.-J. (2001). «La pratique réflexive, pour une cadre de référence partagé par les acteurs de la formation ». Recherche et formation, $n^{\circ} 36$, p. 29-42.

LAPAIRE J-R. (2016). «Préface». In I. Capron Puozzo, La créativité en éducation et en formation. Perspectives théoriques et pratiques. Louvain-La-Neuve : De Boeck, p.7-11.

LEUBA D., DIDIER J, PERRIN N., PUOZZO I. \& VANINI DE CARLO K. (2012). «Développer la créativité par la conception d'un objet à réaliser. Mise en place d'un dispositif de Learning Study dans la formation des enseignants ». Éducation et francophonie, $\mathrm{n}^{\circ} 2$, p. 177-193.

LUBART T. (2010). Psychologie de la créativité. Paris : Armand Colin, $2^{e}$ éd.

MARCEL J.-F., DUPRIEZ V., PERISSET BAGNOUD D. \& TARDIF M. (dir.) (2007). Coordonner, collaborer, coopérer. Bruxelles: De Boeck.

MIALARET G. (1996). «Savoirs théoriques, savoirs scientifiques et savoirs d'action en éducation ». In J.-M. Barbier, Savoirs théoriques et savoirs d'action. Paris : PUF, p. 161-187.

MORISSE M. (2014). «Les dimensions réflexive et professionnalisante de l'écriture : quelques considérations épistémologiques, théoriques et méthodologiques». In M. Morisse \& L. Lafortune, L'écriture réflexive. Objet de recherche et de professionnalisation. Québec : Presses de I'Université du Québec, p. 9-29.

MORISSE M. \& LAFORTUNE L. (dir.) (2014). L'écriture réflexive. Objet de recherche et de professionnalisation. Québec : Presses de I'Université du Québec.

PAQUAY L. (1994). «Vers un référentiel de compétences professionnelles de l'enseignant». Recherche et formation, $n^{\circ} 16$, p. $7-38$.

PERRENOUD P. (2001). Développer la pratique réflexive dans le métier d'enseignant : professionnalisation et raison pédagogique. Issy-les-Moulineaux : ESF.

PIOT T. (2012). «Le langage, organisateur et instrument de la réflexivité professionnelle des enseignants». In. M. Tardif, C. Borgès \& A. Malo, Le virage réflexif en éducation. Où en sommes-nous 30 ans après Schön? Bruxelles : De Boeck, p. 92-105.

ROBINSON K. (2011). Out of our minds: Learning to be creative. West Sussex : Capstone.

SAINT-ARNAUD Y. (2001). "La réflexion-dans-l'action : un changement de paradigme». Recherche et formation, $n^{\circ} 36$, p. 17-27.
SAUSSEZ F., EWEN N. \& GIRARD J. (2001). «Au cœur de la pratique réflexive, la conceptualisation? » Recherche et formation, $\mathrm{n}^{\circ} 36$, p.69-87.

SCHÖN D. (1983). The reflective practitioner. New York : Basic Books.

SCHÖN D. (1994). Le praticien réflexif. À la recherche du savoir cache dans l'agir professionnel. Montréal : Les Éditions logiques.

STERNBERG R. J \& KAUFMAN J. C (2010). «Constraints on Creativity: Obvious and not So Obvious». In J. C. Kaufman \& R. J. Sternberg (dir.), The Cambridge handbook of creativity. Cambridge : Cambridge University Press, p. 467-482.

TARDIF M., BORGÈS C. \& MALO A. (dir.) (2012). Le virage réflexif en éducation. Où en sommes-nous 30 ans après Schön? Bruxelles : De Boeck.

TARDIF M., MARCEL J.-F., PERISSET D. \& PIOT T. (dir.) (2017). L'organisation du travail des acteurs scolaires. Points de repères sur les évolutions au début du XXI siècle. Québec : Presses de l'Université.

TRIQUET É. (2007). «Élaboration d'un récit de fiction et questionnement scientifique au musée». Aster, $\mathrm{n}^{\circ} 44$, p.107-134.

VANASSE G.-G. \& GAUDREAULT M. N. (2004). «Écriture créative et plaisir d'apprendre». Didáctica (Lengua y Literatura), $\mathrm{n}^{\circ} 16$, p. $235-250$.

VANHULLE S. (2002). «La manipulation créative des connaissances par l'écriture, entre implication et distance». Spirale-Revue de recherches en éducation, $\mathrm{n}^{\circ} 29, \mathrm{p} .123-143$.

VANHULLE S. (2005). «Écriture réflexive et subjectivation de savoirs chez les futurs enseignants ». Nouveaux cahiers de la recherche en éducation, vol. 8, $\mathrm{n}^{\circ} 1, \mathrm{p} .41-63$.

VANINI DE CARLO K. \& CLERC-GEORGY A. (2011). «Écriture de fictions scientifiques : un outil d'intégration des savoirs en formation ». Prismes. Revue pédagogique HEP Vaud, n०15, p. 30-31.

VIAU R. (2009). La motivation en contexte scolaire. Bruxelles: De Boeck \& Larcier, $2^{\mathrm{e}}$ éd.

VYGOTSKI L. ([1930] 2004). «Imagination and creativity in childhood ». Journal of Russian and East European Psychology, vol. 42, n¹, p.7-97.

WENTZEL B. (2015). «Questions de subjectivité en formation professionnelle des enseignants». Recherche et formation, n॰80, p. 17-32.

WOODS P. (2001). «Creative Literacy». In A. Craft, B. Jeffrey \& M. Leibling, Creativity in Education. Londres : Continuum, p. 62-79. 
Tableau 1. Synopsis du dispositif de formation. Planification pour la rédaction de l'écriture de fiction scientifique

\begin{tabular}{|c|c|}
\hline Séances & Contenus \\
\hline Séance 1 & $\begin{array}{l}\text { Présentation des objectifs et critères de formation de la 3e année; } \\
\text { Présentation des travaux à réaliser : écriture de fiction scientifique, méta-texte, présenta- } \\
\text { tion orale du bilan de formation, dossier de certification; } \\
\text { Présentation des enjeux de l'écriture de fiction scientifique et de la planification des } \\
\text { tâches; } \\
\text { Présentation de } 2 \text { documents considérés comme des aides à exploiter uniquement si } \\
\text { besoin sur les différents types de genre littéraire pour construire une fiction et la grille } \\
\text { d'auto-évaluation du récit; } \\
\text { Lecture d'un exemple de fiction scientifique (les autres textes sont à lire à la maison); } \\
\text { Lecture du texte de Prismes de Vanini De Carlo et Clerc-Georgy (2011). } \\
\text { Consigne pour S2: apportez le matériel des cours suivis pendant la formation ainsi que les } \\
\text { pièces réalisées. }\end{array}$ \\
\hline Séance 2 & $\begin{array}{l}\text { Prendre le matériel de chaque année, déterminer pour les cours de } 1 \text { re et de } 2 \text { e année les } \\
\text { objets de formation étudiés et les définir; } \\
\text { Brainstorming collectif : énumérer les objets de formation, les définir de manière consen- } \\
\text { suelle en se référant à des sources théoriques; } \\
\text { Écriture individuelle sur l'objet de formation choisi (définition, description et références } \\
\text { théoriques). } \\
\text { Consigne pour le travail à distance : laissez vagabonder le concept dans votre imagination. }\end{array}$ \\
\hline $\begin{array}{l}\text { Travail à distance } \\
\text { Délai d'envoi : } \\
2 \text { décembre } 2015\end{array}$ & $\begin{array}{l}\text { Identification du genre de la narration; } \\
\text { Élaboration de la trame narrative (choix du personnage principal, des personnages secon- } \\
\text { daires, de l'objet de la quête, etc.); } \\
\text { Envoi à la formatrice : 1) du concept de formation choisi défini et expliqué de la manière la } \\
\text { plus exhaustive possible, } 2 \text { ) de la trame narrative sous forme de schéma. }\end{array}$ \\
\hline Séance 3 & $\begin{array}{l}\text { Présentation des objectifs du méta-texte; } \\
\text { Accompagnement dans l'écriture; } \\
\text { Point de la situation : difficultés en formation? sur le terrain? }\end{array}$ \\
\hline Séance 4 & $\begin{array}{l}\text { Rédaction de l'écriture de fiction scientifique; } \\
\text { Échange collectif : présentation de l'évolution de l'histoire et des difficultés rencontrées. }\end{array}$ \\
\hline $\begin{array}{l}\text { Travail à distance } \\
\text { Délai d'envoi : } \\
16 \text { mars } 2016\end{array}$ & Envoyez une première esquisse de votre texte à la formatrice pour un retour formatif. \\
\hline Séances 5 et e 6 & Séance bilan : lecture des récits de fiction scientifique et bilan de formation \\
\hline
\end{tabular}




\begin{tabular}{|c|c|c|c|}
\hline Nom & Résumé des fictions scientifiques & $\begin{array}{l}\text { Concepts et auteurs } \\
\text { convoqués }\end{array}$ & Créativité \\
\hline $\begin{array}{l}\text { Chrystal et } \\
\text { Marika }\end{array}$ & $\begin{array}{l}\text { Disparition inquiétante. C'est l'histoire d'une sortie } \\
\text { d'école d'une classe de } 6 \text { h dans un musée au nom } \\
\text { mystérieux. Soudain, durant la visite, l'alarme } \\
\text { sonne et les élèves se précipitent à l'extérieur. Mais } \\
\text { Hubert manque à l'appel. Le jeune garçon } \\
\text { découvre alors le mystérieux secret du musée. }\end{array}$ & $\begin{array}{l}\text { Aucun concept n'est } \\
\text { explicitement trouvé } \\
\text { dans I'histoire }\end{array}$ & $\begin{array}{l}\text { La fiction scienti- } \\
\text { fique n'est pas adap- } \\
\text { tée au genre de } \\
\text { l'écriture créative }\end{array}$ \\
\hline Marguerite & $\begin{array}{l}\text { Pensée divergente et pensée convergente, un chemin } \\
\text { incontournable vers la création innovante. C'est l'his- } \\
\text { toire de Yves, le designer, créatif qui se lance dans } \\
\text { l'écriture d'une histoire destinée à sa fille. Le père } \\
\text { l'emmène dans le pays de la " para-fantaisie [...] où } \\
\text { toutes les idées ont leur place». }\end{array}$ & $\begin{array}{l}\text { Les concepts développés } \\
\text { sont la pensée diver- } \\
\text { gente/convergente et } \\
\text { l'innovation }\end{array}$ & $\begin{array}{l}\text { La fiction scienti- } \\
\text { fique n'est pas adap- } \\
\text { tée au genre de } \\
\text { l'écriture créative }\end{array}$ \\
\hline Florent & $\begin{array}{l}\text { Trois colocataires, enseignantes d'Activités créa- } \\
\text { trices et manuelles, vivent dans le même apparte- } \\
\text { ment et travaillent au sein du même établisse- } \\
\text { ment. Un petit concours s'ouvre autour de la } \\
\text { réalisation d'un porte-crayons dans l'établisse- } \\
\text { ment avec un voyage comme prix. Chaque ensei- } \\
\text { gnante travaille différemment avec ses élèves pour } \\
\text { leur faire réaliser l'objet : Missif est sur une } \\
\text { approche transmissive, Viorisite sur une démarche } \\
\text { béhavioriste et Tiviste sur un enseignement } \\
\text { constructiviste. Dans les trois cas, les élèves } \\
\text { avancent difficilement. Après deux mois, les élèves } \\
\text { sont démotivés et n'ont pas fini leur porte-crayons. } \\
\text { Morale de l'histoire : la mise en œuvre d'une seule } \\
\text { méthode n'est pas suffisante pour réussir. C'est } \\
\text { l'éclectisme qui doit être privilégié. }\end{array}$ & $\begin{array}{l}\text { Les concepts sont deux } \\
\text { théories d'apprentissages } \\
\text { (le béhaviorisme et le } \\
\text { constructivisme) et la } \\
\text { méthode transmissive }\end{array}$ & $\begin{array}{l}\text { La fiction scienti- } \\
\text { fique n'est pas adap- } \\
\text { tée au genre de } \\
\text { l'écriture créative }\end{array}$ \\
\hline Marco & $\begin{array}{l}\text { La créativité au service de la justice. Cette nouvelle } \\
\text { policière raconte l'enquête de l'inspecteur Eugène } \\
\text { Crintiff sur le meurtre de Marcel, victime du tueur } \\
\text { de glace encore non arrêté. Après de nombreux } \\
\text { rebondissements, l'inspecteur démasque le tueur. }\end{array}$ & $\begin{array}{l}\text { Aucun concept n'est } \\
\text { explicitement trouvé } \\
\text { dans I'histoire }\end{array}$ & $\begin{array}{l}\text { La fiction scienti- } \\
\text { fique n'est pas adap- } \\
\text { tée au genre de } \\
\text { l'écriture créative }\end{array}$ \\
\hline Fiona & $\begin{array}{l}\text { De fil en aiguille. Pétronille, I'aiguille, et Théophile, } \\
\text { le fil, sont deux amis; Pétronille rêve de connaître } \\
\text { l'origine de son innovation. Un matin, elle propose } \\
\text { à son ami un voyage dans le passé pour rencontrer } \\
\text { ses ancêtres aiguilles. À leur retour Pétronille y } \\
\text { gagne en confiance en soi et est plus en symbiose } \\
\text { avec Théophile. }\end{array}$ & $\begin{array}{l}\text { L'aiguille à coudre, son } \\
\text { histoire et les techniques } \\
\text { concernent des savoirs } \\
\text { appris dans les branches } \\
\text { techniques de la forma- } \\
\text { tion, et notamment la } \\
\text { couture }\end{array}$ & $\begin{array}{l}\text { La fiction scienti- } \\
\text { fique n'est pas adap- } \\
\text { tée dans le sens qu'il } \\
\text { n'y a pas de fusion } \\
\text { entre les univers } \\
\text { conceptuel et } \\
\text { fictionnel }\end{array}$ \\
\hline Berthe & $\begin{array}{l}\text { Tout fout l'camp ou presque. C'est l'histoire d'une } \\
\text { jeune fille de } 11 \text { ans qui entre en } 8 \text { e avec un par- } \\
\text { cours scolaire difficile qu'elle décrit. Le premier } \\
\text { cours de couture commence et au moment où } \\
\text { l'enseignant prononce le mot tricot, son hurlement } \\
\text { la fait aller dans le passé. L'enseignante du passé se } \\
\text { met à raconter l'histoire du tricot, puis fait tricoter } \\
\text { la classe et guidant chaque mouvement. Puis le } \\
\text { personnage principal revient à son époque et sait } \\
\text { désormais coudre. }\end{array}$ & $\begin{array}{l}\text { Le tricot et l'enseigne- } \\
\text { ment de la couture sont } \\
\text { des objets de savoir } \\
\text { appris à la fois dans la } \\
\text { branche technique de la } \\
\text { couture, mais aussi en } \\
\text { didactique des activités } \\
\text { créatrices et manuelles }\end{array}$ & $\begin{array}{l}\text { La fiction scienti- } \\
\text { fique n'est pas adap- } \\
\text { tée dans le sens qu'il } \\
\text { n'y a pas de fusion } \\
\text { entre les univers } \\
\text { conceptuel et } \\
\text { fictionnel }\end{array}$ \\
\hline
\end{tabular}




\begin{tabular}{|c|c|c|c|}
\hline Nom & Résumé des fictions scientifiques & $\begin{array}{l}\text { Concepts et auteurs } \\
\text { convoqués }\end{array}$ & Créativité \\
\hline Jade & $\begin{array}{l}\text { "La créativité est contagieuse, faites la tourner» } \\
\text { Albert Einstein.L'histoire est celle des castors. } \\
\text { L'histoire débute sur la description d'une journée } \\
\text { ordinaire d'un castor. Jasmin, le personnage princi- } \\
\text { pal, est un jeune castor rêveur. Un jour, des } \\
\text { hommes se mettent à détruire la forêt où le jeune } \\
\text { castor vivait avec sa tribu qui se réfugie alors dans } \\
\text { une grotte. Après plusieurs jours, Jasmin essaie de } \\
\text { motiver les troupes. Une organisation se met en } \\
\text { place pour reconstruire les habitats. }\end{array}$ & $\begin{array}{l}\text { Le concept est celui de la } \\
\text { créativité }\end{array}$ & $\begin{array}{l}\text { La fiction scienti- } \\
\text { fique n'est pas adap- } \\
\text { tée dans le sens qu'il } \\
\text { n'y a pas de fusion } \\
\text { entre les univers } \\
\text { conceptuel et } \\
\text { fictionnel }\end{array}$ \\
\hline Marc & $\begin{array}{l}\text { L'importance du cahier des charges et du modèle } \\
\text { C-R-S lors de la construction d'une mégapole par des } \\
\text { fourmis champignonnistes. L'histoire se base sur un } \\
\text { dialogue entre un papa fourmi et son fils. Ce der- } \\
\text { nier lui enseigne comment construire une } \\
\text { mégalopole. }\end{array}$ & $\begin{array}{l}\text { Le modèle didactique } \\
\text { enseigné «conception- } \\
\text { réalisation-socialisation » } \\
\text { en didactique est le } \\
\text { concept traité }\end{array}$ & $\begin{array}{l}\text { La fiction scienti- } \\
\text { fique n'est pas adap- } \\
\text { tée dans le sens qu'il } \\
\text { n'y a pas de fusion } \\
\text { entre les univers } \\
\text { conceptuel et } \\
\text { fictionnel }\end{array}$ \\
\hline Joseline & $\begin{array}{l}\text { L'histoire est celle d'une émission télévisée } \\
\text { «Masterprof ». C'est le moment de la finale. Les } \\
\text { deux enseignants s'affrontent en enseignant une } \\
\text { discipline qui n'est pas la leur à un public d'élèves } \\
\text { qu'ils ne connaissent pas. Les situations didac- } \\
\text { tiques sont bien décrites en fonction des théories } \\
\text { dans un registre humoristique : transmissif d'un } \\
\text { côté, expérientiel de l'autre. Au moment de } \\
\text { connaître le vainqueur, pour des raisons budgé- } \\
\text { taires, le programme s'interrompt brutalement. }\end{array}$ & $\begin{array}{l}\text { Les concepts sont les } \\
\text { théories d'apprentissage } \\
\text { (le béhaviorisme et la } \\
\text { méthode Freinet) }\end{array}$ & $\begin{array}{l}\text { La fiction scienti- } \\
\text { fique n'est pas adap- } \\
\text { tée, car il manque } \\
\text { les références au } \\
\text { savoir scientifique }\end{array}$ \\
\hline Philippe & $\begin{array}{l}\text { Cette courte pièce de théâtre est un dialogue } \\
\text { entre deux mamans qui parlent du cadeau de la } \\
\text { fête des Mères réalisé durant le cours des Activités } \\
\text { créatrices et manuelles. }\end{array}$ & $\begin{array}{l}\text { Les concepts sont la } \\
\text { conception de projet et } \\
\text { la créativité (divergence, } \\
\text { convergence) }\end{array}$ & $\begin{array}{l}\text { La fiction scienti- } \\
\text { fique n'est pas adap- } \\
\text { tée, car il manque } \\
\text { les références au } \\
\text { savoir scientifique }\end{array}$ \\
\hline Lucie & $\begin{array}{l}\text { Ce texte est un discours inaugural des premières } \\
\text { «commodités» de Versailles. }\end{array}$ & $\begin{array}{l}\text { Les concepts sont la créa- } \\
\text { tivité, l'innovation (consi- } \\
\text { dérée comme une créati- } \\
\text { vité avec un grand C), } \\
\text { l'approche multivariée } \\
\text { (malheureusement } \\
\text { Lubart n'est pas men- } \\
\text { tionné), les macro- } \\
\text { processus créatifs et } \\
\text { l'imagination. } \\
\text { Les références historiques } \\
\text { sont bien présentes, mais } \\
\text { le savoir scientifique n'est } \\
\text { pas rattaché à un auteur. } \\
\text { Néanmoins, le lien entre } \\
\text { créativité et innovation } \\
\text { est très bien construit }\end{array}$ & $\begin{array}{l}\text { La fiction scienti- } \\
\text { fique répond aux } \\
\text { contraintes et est } \\
\text { donc créative }\end{array}$ \\
\hline
\end{tabular}




\begin{tabular}{|c|c|c|c|}
\hline Nom & Résumé des fictions scientifiques & $\begin{array}{l}\text { Concepts et auteurs } \\
\text { convoqués }\end{array}$ & Créativité \\
\hline Nadia & L'histoire est celle de l'invention du stylo Bic. & $\begin{array}{l}\text { Les concepts de facteurs } \\
\text { cognitifs (la pensée diver- } \\
\text { gente) et conatifs (la per- } \\
\text { sévérance, la tolérance à } \\
\text { l'ambiguïté, l'ouverture à } \\
\text { de nouvelles expériences, } \\
\text { individualisme, prise de } \\
\text { risque) de la créativité } \\
\text { sont décrits à travers } \\
\text { l'histoire du stylo Bic }\end{array}$ & $\begin{array}{l}\text { La fiction scienti- } \\
\text { fique répond aux } \\
\text { contraintes et est } \\
\text { donc créative }\end{array}$ \\
\hline Lisa & $\begin{array}{l}\text { Gros soucis au pays du père Noël. Le } 23 \text { décembre, } \\
\text { l'atelier des cadeaux, censé être plein de lutins en } \\
\text { action, est désert. Le père Noël, étonné, va les } \\
\text { réveiller. Il les trouve alors frigorifiés et cloués au } \\
\text { lit. Ils interpellent alors les fées pour l'aider à les } \\
\text { réchauffer. L'une des fées à l'idée de confectionner } \\
\text { des chaussons et des bonnets en feutrage pour les } \\
\text { réchauffer. Tous se mettent au travail en confec- } \\
\text { tionnant ces derniers et le Noël des enfants est } \\
\text { sauvé. }\end{array}$ & $\begin{array}{l}\text { Le concept traité est celui } \\
\text { du feutrage : I'histoire et } \\
\text { sa technique }\end{array}$ & $\begin{array}{l}\text { La fiction scienti- } \\
\text { fique répond aux } \\
\text { contraintes et est } \\
\text { donc créative }\end{array}$ \\
\hline $\begin{array}{l}\text { Isabelle et } \\
\text { Brigitte }\end{array}$ & $\begin{array}{l}\text { Silence, je pense. Le texte se présente comme un } \\
\text { extrait de journal intime, rédigé par Valentin, } \\
13 \text { ans. Le personnage principal y raconte ses } \\
\text { aventures à l'école où il est considéré comme élève } \\
\text { en difficulté. Seule la branche des Activités créa- } \\
\text { trices et manuelles lui plaît et il s'investit dans } \\
\text { cette dernière en devenant un élève créatif. }\end{array}$ & $\begin{array}{l}\text { Le concept de la créati- } \\
\text { vité est traité dans ce } \\
\text { texte }\end{array}$ & $\begin{array}{l}\text { La fiction scienti- } \\
\text { fique répond parfai- } \\
\text { tement aux } \\
\text { contraintes et est } \\
\text { donc créative }\end{array}$ \\
\hline
\end{tabular}

